\title{
Playfully Learning Visual Perspective Taking Skills with Sifteo Cubes
}

\author{
Luc Geurts, Vero Vanden Abeele, Kevin Van Keer, Ruben Isenborghs \\ KU Leuven - e-Media Lab \\ Andreas Vesaliusstraat 13, 3000 Leuven, Belgium \\ \{luc.geurts, vero.vandenabeele\}@kuleuven.be, \\ $\{$ kevin.vankeer, ruben.isenborghs\}@student.kuleuven.be
}

\begin{abstract}
In this paper we describe the design, development and testing of two computer games using Sifteo Cubes that help children to train their visual perspective taking (VPT) skills, i.e. the ability to see the world from another person's perspective. The challenge was to design an enjoyable and usable game that takes into account the huge variability in the perspective taking skills within the target group (preschoolers at the age of five, and older children with learning disabilities). Sifteo Cubes can be considered as digital or intelligent manipulatives that are often used in instruction. We advocate that these type tangible objects can help children performing VPT related tasks since they allow for actions in the real world that aid their thinking. Pre-test and post-test results revealed a short term learning effect on VPT skills after playing the two games.
\end{abstract}

\section{Author Keywords}

Sifteo Cubes; digital manipulatives; tangible interaction; serious games; visual perspective taking

\section{ACM Classification Keywords}

H.5.2. Information interfaces and presentation (e.g., HCI): User Interfaces - Input devices and strategies.

\section{INTRODUCTION}

The last decade has been characterized by the emergence of novel interaction technologies. Well-known commercial examples are Nintendo Wii, PlayStation Move and Microsoft Kinect. These technologies offer the opportunity to design new types of games, based on physical motion, and a have broadened the application area to 'serious games' and more particularly games for health; adults play exertion games to maintain their physical conditions, seniors play motion based games for fall rehabilitation, and children play these type of games to improve their psychomotor skills.

Permission to make digital or hard copies of all or part of this work for personal or classroom use is granted without fee provided that copies are not made or distributed for profit or commercial advantage and that copies bear this notice and the full citation on the first page. Copyrights for components of this work owned by others than ACM must be honored. Abstracting with credit is permitted. To copy otherwise, or republish, to post on servers or to redistribute to lists, requires prior specific permission and/or a fee. Request permissions from Permissions@acm.org.

CHI PLAY'14, October 19 - 22 2014, Toronto, ON, Canada

Copyright 2014 ACM 978-1-4503-3014-5/14/10 ..\$15.00.

http://dx.doi.org/10.1145/2658537.2658706
Also more graspable or tangible objects have been suggested as a way to extend interaction [3,9]. When tangible interfaces make use of multiple interactive objects, the input is called space-multiplexed ${ }^{1}$; different tangibles, dispersed over the environment, can represent different actions or hold different information. These different tangible objects can come in different shapes, sizes and locations [16], which supports epistemic actions. Epistemic actions are unplanned actions that distribute some of the work of problem solving to the environment [6]. Epistemic actions do not directly bring a person closer to its goal but rather change the world in order to simplify the problemsolving task [1]. Clearly, space-multiplexed tangibles allow for epistemic actions: tangibles can be moved around; pieces can be shifted, individually or in group; objects can be touched or pointed at; blocks can be partitioned and recomposed in subsets, etc. Epistemic actions precede pragmatic actions, the latter directly bring the child closer to its goal, i.e. solving the problem. The division between epistemic versus pragmatic actions also underpins Physically Distributed Learning (PDL) [10]. Yet PDL emphasizes that actions and interpretations coevolve; actions and interpretations develop each other, and over time people develop stable ideas and solve problems using mental strategies.

In this manner, space-multiplexed tangibles also particularly lend themselves to PDL. Not surprisingly, the theory of Physically Distributed Learning has been built alongside research involving physical manipulatives for mathematics learning [11]. Manipulatives are concrete materials aimed to benefit children's learning $[2,12,13]$.

Space-multiplexed tangibles could be considered digital manipulatives [15], and possibly can provide several benefits compared to popular platforms such as in recent years tablets. Intelligent manipulatives blur the boundary between the real and the digital world, and exploit the sense of touch as opposed to e.g. touch interaction: users can feel edges, curves, and ridges; different textures, pliability,

1 Space-multiplexed interfaces are juxtaposed to timemultiplexed interfaces such as GUIs and computer mice, where a user can only interact with one object at a time. 
temperature; distribution of weight; as opposed to the "glass plate" that exists between the user and the digital content in a tablet [20].

A recent popular game platform are the Sifteo Cubes that can be considered as intelligent manipulatives as well. Each cube has a touch sensitive display and contains sensors to detect motion and identify adjacent cubes. This leads to several interaction modes such as tilting, flipping, shaking, neighboring and pressing, as illustrated in Figure 1.

Several researchers have explored the potential of the Sifteo Cubes in various application areas. Hunter et al. presented two language-learning applications for children between 4 and 7 years old. The focus of one application is to teach children spatial concepts and basic sentence-construction skills through creative play, while the other game was designed to teach vocabulary and reading [8]. CogCubed (www.cogcubed.com) developed a diagnostic tool for testing for symptoms of Attention Deficit Hyperactivity Disorder [6]. Vandermaesen et al. presented 'PhysiCube', a rehabilitation system that provides motivating physical training for the upper limbs. Instead of manipulating daily objects, the patients play a game by lifting and reaching to Sifteo Cubes [19]. Pillias et al. developed and tested a collaborative game and advocate that the cubes' properties "provide players with more freedom and relatedness, while helping to create an easy-to-learn and customizable gameplay [14]."

Another application area where Sifteo Cubes might be of particular use is Visual Perspective Taking (VPT). Visual Perspective Taking is the ability to see the world from another person's perspective, taking into account what they see and how they see it [4,5,17] (see Figure 2). Children gradually acquire this skill during kindergarten and are assumed to have mastered this skill when they leave preschool for primary education. However, many five- or six-year olds still have difficulties identifying their own left and right, and identifying another person's left and right presents an even greater challenge. In this case, it might be beneficial to see a psychomotor therapist. Typically, psychomotor therapists rely on exercises and drawings on paper for the children to train these skills. Generally, children find these paper-based exercises quite boring. Incorporating these exercises in a game is likely to increase the children's motivation.

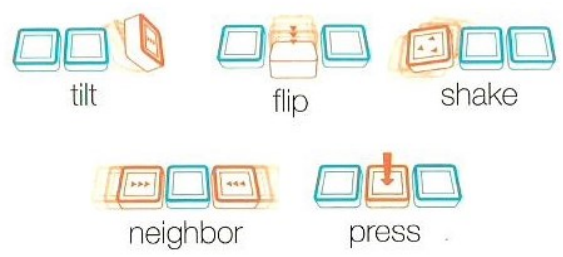

Figure 1 Possible interaction modes with Sifteo Cubes

\section{Research question}

The main objective of our project was to explore the benefits that digital manipulatives, and in particular Sifteo Cubes, might offer for young children learning or improving VPT skills. The challenge was to design games that are enjoyable and usable and that train VPT in fiveyear-olds.

As aforementioned, the main motivation to choose the Sifteo platform to train VPT, is Sifteo system's potential to enable physically distributed learning. While exercises on paper or tablet still require the child to think and operate in a 2D world, cubes can be distributed and manipulated in 3D space, by grasping, lifting and replacing them. These actions are very natural to these children, so the necessary manipulations are supposedly intuitive, they do not need to be learned. Also, as opposed to paper exercises, children can perform epistemic actions: they can take and replace cubes in order to help their thinking. Finally, digital manipulatives offer the opportunity to create interactive systems that provide immediate feedback to the player's actions. In conclusion, we postulate that games can be designed that can be learned very quickly, so that most mental effort has to go to the real challenge (i.e. VPT).

In the next section we will first discuss our iterative design process, and the two games we developed. Next, we will present and discuss the results of an experiment in which we investigated the usability of the games, the response from the players, and possible short term learning effects.

\section{DESIGN}

As is evident when designing games for young children, we adopted an iterative player-centered approach [18]. There is indeed a large discrepancy between the cognitive abilities of the game designers and those of the target group, resulting in a risk that incorrect preconceptions influence the game design.

Therefore, a psychomotor therapist was involved in this project. Her job consists of helping children with learning disabilities, varying from assisting in reading, writing, and mathematics, problem solving and performing daily activities. Her major contributions in this study were giving feedback on initial game concepts and assisting in and commenting on the intermediate user studies with children with learning disabilities. In addition to the psychomotor therapists, six children were involved as well, aged between 5 and 7 . Three of these children were clients of the psychomotor therapist having problems with VPT, whereas the three other children were recruited via relatives of the researchers, and did not have known VPT-related problems.

Over a period of 7 weeks, game concepts and later interactive prototypes were designed and evaluated by the therapists and these children during formative usability tests. From these iterations we derived the following guidelines for training VPT skills. 

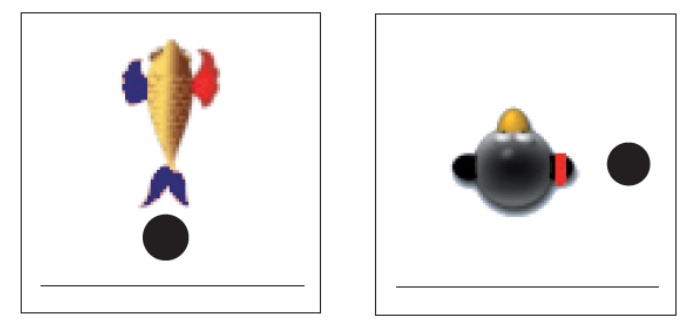

Figure 2 Visual perspective taking refers to the ability to identify another person's left, right, front or back. A typical question in a test would be: "If you were the fish, where would in the black dot be? In front of you, behind you, at your left, or at your right?"

\section{General Guidelines for training Visual Perspective Taking Skills}

The goal of the games is to train the child in its VPT skills. More specifically, the child should learn to identify another person's left, right, front and back. The challenge is twofold: some of these children already have difficulties identifying their own left and right, and taking the perspective of another person makes the task even more challenging. Therefore, the game should add no additional complexity or other problem solving task, but focus on maintaining a high motivation of the child to keep on training his or her VPT skills. In consultation with the therapist and children, the following requirements were formulated:

1. Difficulty levels should increase progressively through all levels, starting with a very basic level where the orientation of the child and the 'other person' (aka the avatar in the game) is identical.

2. When mistakes are made, immediate feedback should be given, with clear information on the error that has been made.

3. The games should include immediate rewards, and few or no punishments should be given, in order to maintain the child's motivation.

4. The children should be able to play the games by themselves, without the aid of someone else.

During the course of the design process we decided to develop and test two games, each exploiting a different interaction style of the Sifteo Cubes. One game, the Fish Game, is mainly based on the neighboring function of the cubes and offers the advantage that the player always has a visual overview on all cubes involved. The other game, the Penguin Game, uses the shake function, but more importantly, in this case, the cubes are distributed in space, at several meters from each other. The idea is that the player has to move through the physical space, and has to perform bodily actions that are related to the challenge at hand. This might enlighten the mental rotation that has to take place when performing a VPT task. In [17] it was indeed concluded that "embodied self-rotations, through which we actively imagine ourselves assuming someone else's position in the world can subserve not only reasoning about where objects are in relation to someone else but also how the objects in their environment appear to them."

\section{Fish Game}

The main goal of the Fish Game is to help the fish to collect candy (see Figure 3, top left cube). The fish appears initially on one of the cubes. A bar of bubbles on the cube's display indicates at which side the player should be. The child is instructed to always place itself at the side with the bubbles.

One or more other cubes show a generic shape (circle, square...). When pressing on one of these cubes, a spoken instruction is given by the fish, e.g. "Put the square at my left side". The player then has to "neighbor" this cube at one of the sides of the fish cube. In case this is done correctly, a candy is revealed, and the fish swims toward it. In case of an incorrect response, no candy is gained, and feedback is given, e.g. "You placed the square at my right side. Please put it at my left side." The cube with the fish is not allowed to be manipulated. An alarming sound will play when the player moves this cube (detected by the cube's accelerometer). The sixth cube shows a box with all the collected candy.

The Fish Game contains five levels, in increasing complexity. There is a "zero" tutorial level that explains all relevant rules of the game. Only when no mistakes are made, the player moves on to the next level. During the first three levels, the fish is oriented in the same direction as the player. Four cubes have to be positioned around the fish in

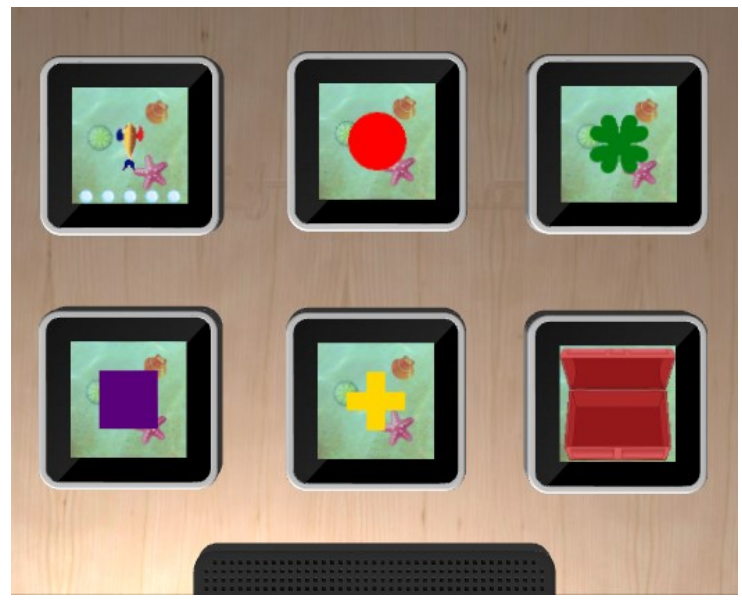

Figure 3 Six cubes are used in the Fish Game. One shows the main character, a fish that has to collect candy. The bubble bar on that cube shows where the player should be located. Four blocks show generic shapes revealing candy when placed at the correct side of the fish' cube. The sixth cube shows a box with all the collected candy. 


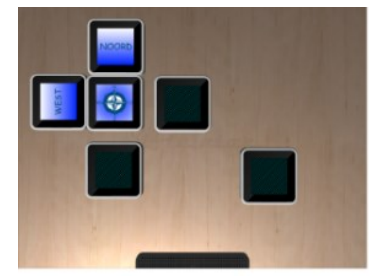

(a)

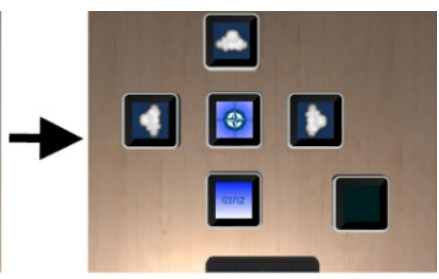

(b)

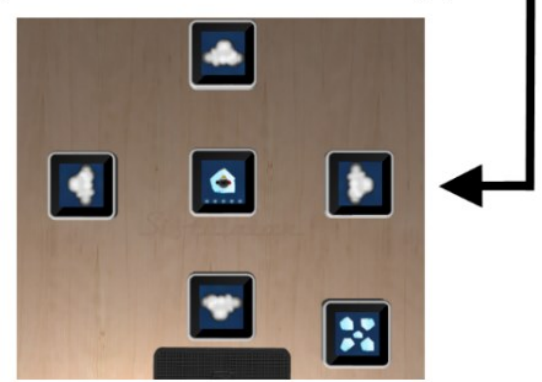

(c)

Figure 4 The cubes of the Penguin game are distributed in space in the four directions (north, east, south, west), at several meters from the middle block with the Penguin. The Penguin tells the player where the bird is hiding (e.g. "at my left"). The birds can be frightened away by shaking the correct cube.

the first level, two times two cubes are to be positioned during the second level (only left and right), four times one cube has to be positioned in the third level (left or right). Hence, the difficulty increases in each level since there are less options to exclude in higher levels. In the last two levels, the fish is oriented with its tail towards its previous cube, so differing from the player's orientation. Level 4 tests on front/back discrimination, level 5 on left/right. Hence, in these last two levels we come across the more challenging Visual Perspective Taking through another's person (in this case a fish) eyes.

\section{Penguin Game}

The main goal of the Penguin Game is to prevent the birds from stealing the Penguin's fish. To set-up the game, five cubes have to be placed in a cross (see Figure 4). The middle one is the Penguin's cube, the four others have to be placed a few meters away in the four directions (north, east, south, and west). Similar to the row of bubbles in the fish game, the player has to be aligned with the row of snowflakes at one side of penguin's cube. The other four cubes initially show a cloud behind which a pestering bird might be hiding. Then the game can start: the player presses the Penguin cube, and hears an instruction from the Penguin, e.g. "The bird is hiding in front of me". The player has to scare away the bird by shaking the correct cube out of the four possible cubes. In case the correct cube is shaken, the cloud will disappear, and the bird will fly away. In the other case, the bird will show up on another cube (the correct one) and steal a fish. A sixth cube shows the total amount of fish. A snowflakes bar on one of the sides of the display indicate at which side the player should be located.

The levels are very similar to the Fish game. This way it is assured that the complexity of the task is identical in each level, and so it allows a fair comparison between both games. The Penguin game has a sixth level though. In this level, the snowflakes bar is positioned at the side pointing to the cube that was last shaken, so it changes after every task. The motivation to include this level is the fact that children are constantly moving around and may arrive from different directions to the cube, ending up at another side.

To conclude, the main difference with the Fish Game is that the player has to move in space, which might be more fun, and more importantly, might encourage the child to perform physical rotations with its own body in order to align with the Penguin (as opposed to mental rotations). On the other hand, the task might be more complex, since the player has no clear overview on all cubes.

\section{Lessons learned}

During the formative user tests we made several observations that lead to improvements in the game design:

1. We observed a huge variability in the children's competences. Some children still had difficulties identifying their own left and right, so even at the lowest level, the game was too difficult. We decided to provide an extra cue by coloring the fish' right fin and the penguin's right wing red (see Figure 2).

2. Initial designs made use of 3D characters. However, the clearest view is the top view, so we chose objects that are easily recognizable from the top. This also ensured a clear indication on the object's orientation.

3. The player should always be located at the correct side of the Fish cube or the Penguin cube, indicated by a bubble bar or a snowflakes bar. Certainly in the first levels, where the orientation of the fish or the penguin is similar to the player, this is an extra help.

4. In the Penguin game we initially changed the position of the snowflakes bar, so that it appeared at the side from which the player returned after shaking a cube. We thought this would a more logical approach but it turned out to confuse the player even more, who was expecting the bar to stay at the same side.

\section{EVALUATION}

\section{Rationale}

In order to evaluate the two games, an experiment was conducted with eight preschoolers (age five years). We wanted to evaluate several aspects. The first question was 
whether the games are enjoyable and that no obstacles prevented game play. Given the great variability in the children's VPT skills at the age of five [5], some children might find the games too difficult. It was also our aspiration to create games that can be played without assistance, and that a few instructions would suffice in order to be able to play the games. A second question was whether the games yield any learning effect, in other words: do the children's VPT skills improve after playing the games? A third question was what strategies children use when playing the game: Do they profit from the tangibility that the games offer? Are epistemic actions observed? Can we see physically distributed learning in that actions and interpretation coevolve? And are these different for the two games? The final question was whether the children have a preference for one of the games and what motives they might have for this preference.

\section{Methodology}

Eight preschoolers participated in the experiments that were conducted in a separate room of their school. None of them participated in the formative tests. After a short introduction by the two experimenters and the goals of the experiment, a pre-test was taken consisting of 10 questions related to VPT (see example in Figure 2). This test was an adaptation of a paper test often used by the psychomotor therapist. Answers were either correct or wrong. The scores would provide insight in the initial level of VPT skills. Then two games were played. In order to compensate for possible ordering effects, four children started with the Fish Game, the other four with the Penguin Game. Each game was explained with the tutorial level (level 0), and help was provided when needed. Then the game was played during 10 minutes without interruption or pauses between the levels, and without any intervention from the experimenter. The number of mistakes were counted, as also the obtained level. Both experimenters observed the children while playing, thereby focusing on the used strategies. After playing the games, a post-test similar to the pre-test was taken in order to investigate a potential learning effect. Note however that we did not include a control group in this

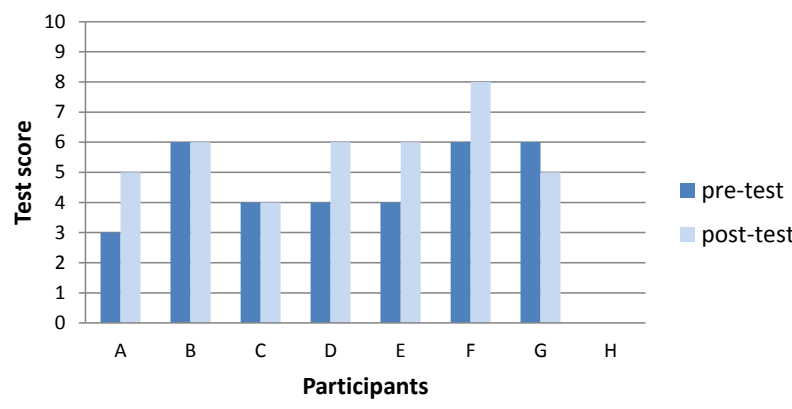

Figure 5 Results for the pre-test and the post-test for all participants. Note that subject $H$ was not able to answer the questions. study, e.g. with children playing a non-VPT related game between the pre-test and the post-test. So, it cannot be concluded that any statistical effect is induced by the games only. Finally, the children were asked which of the two games they preferred and why.

\section{Results}

Figure 5 shows the results of the pre-tests (dark bars). Scores vary from $0 / 10$ (subject $H$ ) to $6 / 10$, illustrating a large variety in initial VPT skills. Subject $\mathrm{H}$ indicated that he had no idea what the answers should be.

On the other hand, all participants were able to play the games. There were no drop-outs, the children kept on playing in upper concentration during 10 minutes. They gave no indication of boredom nor frustration, even though they had to perform difficult tasks. Figure 6 shows the obtained levels for all participants for both games. Two children completed all levels of one game. There is also a trend that the children were more successful in the second game, which is probably due to a higher acquaintance with the Sifteo cubes and the similar game mechanics in both games. Note also that subject $\mathrm{H}$, who indicated not to be able to do the paper test, was able to reach at least level 4 in our games. We can conclude from these results that both games are playable, regardless the initial VPT ability.

The number of mistakes was very similar for both games. Across all participants, 19\% of the instructions yielded an incorrect answer for the Fish game, while this number was $18 \%$ for the Penguin game. As also indicated by the children, the games had equal difficulty, even though they have different interaction styles.

A comparison between the results of the pre-test and the post-test revealed a moderate but significant effect (Figure 5). A one-tailed paired t-test revealed a significance difference between the scores $(p=0.0437 \%)$. This might imply that the children indeed improved their VPT skills by playing the game, and were able to make the transfer to the paper test. However, as stated before, since no control group was included, this increase might be due to other effects, such as practice effects of the tests.

Observations during the playing confirmed that the children took their time to "think with their body" about the actions they had to perform. In case the fish' or penguin's orientation was different from the players', they tend to spontaneously turn their head or their whole body (in case of the Penguin Game), to align their orientation with that of the avatar. This phenomenon manifested itself more in the Penguin Game than in the Fish Game, so this could be an indication that provoking whole-body action has an advantage. In the Fish Game, children moved cubes around in different positions, before neighboring them to the fish cube. These movements indicate that they spontaneously performed epistemic actions. 
No agreement was observed in the children's preference. Three children preferred the Fish Game, because they thought it was easier to play, or for less relevant reasons ("I like candy, so I like the Fish Game more.”). Three other children preferred the Penguin Game because they liked moving around. The two remaining children could not make up their mind. This confirms that players should not be treated as a monolithic group and that it might be beneficial to design different games depending on the player type, instead of adopting a one-size-fits-all approach.

\section{DISCUSSION}

The results seem to suggest that tangible games do offer several advantages compared to traditional exercises. Even children performing poorly on the paper test were able to complete several levels of the games, thereby confirming that they were able to answer VPT related questions correctly. Although a fair comparison between the number of mistakes on the paper test and those on the games is quite impossible, there is a trend that less mistakes were made when playing the game. This is an encouraging observation, for it might have turned the other way around. Indeed, one could argue that embedding challenging tasks in a game environment adds complexity, since the player should not only focus on the task at hand, but also to other game-related aspects (e.g. knowing which cube to handle, when, and how). An obvious explanation for the better performance with the Sifteo Cubes is the incorporation of feedback in the games. This way children were able to correct their mistakes, a possibility not incorporated in the paper test. A second explanation is the more ecological setting of the game. Instead of having to make judgments based on static drawings on a 2D paper surface, the children operated in a more realistic setting, with $3 \mathrm{D}$ objects that were presented in a $3 \mathrm{D}$ environment. Note that even the child (participant $\mathrm{H}$ ) that was unable to perform the paper test, could solve the VPT related questions when playing.

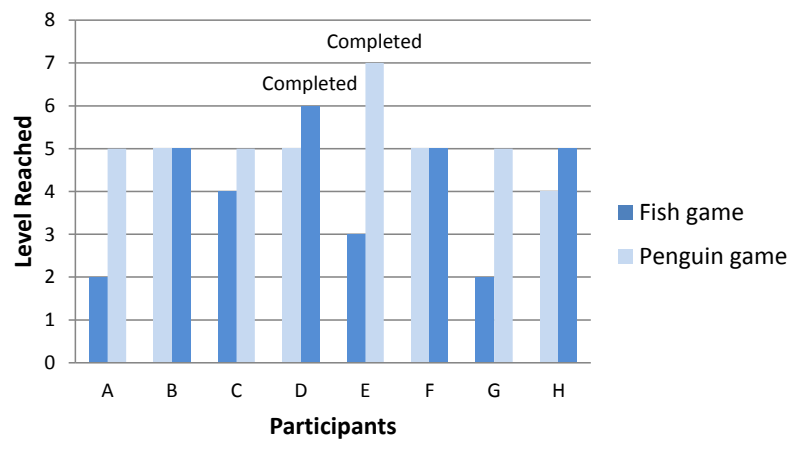

Figure 6 Levels reached after 10 minutes of playing for all participants, and for both games. Subject $D$ completed all levels of the Fish Game (indicated as non-existing level 6), subject $\mathbf{E}$ completed all levels of the Penguin Game.
Also the fact that epistemic actions were observed probably contributes to the good performance of the children. In the case of the Penguin Game, such an epistemic action could be the player turning himself in the direction the penguin is looking. This action does not actually bring the player to the correct solution, but lightens the cognitive burden of replacing himself in the place of the penguin. These epistemic actions were used more in the Penguin Game than in the Fish Game, however, the mistake rate is about the same in both games. A possible cause of this is that the player in the Fish Game always has an overview on all cubes. This visual overview is lacking in the Penguin Game, which could counteract the beneficial effect of the epistemic actions. A hybrid approach, which would e.g. include a display with an overview in the Penguin Game, could then combine the best of both worlds [7].

Another encouraging observation were the short-term learning effects. This suggests that the children were able to transfer the skills they learned by playing the games (in a $3 \mathrm{D}$ environment) to the tasks at hand on the (2D) paper test. However, we acknowledge that the small sample (8 children) and the lack of a control group necessitates caution with respect to broad claims. More research with more children is necessary to strengthen our findings. In addition, it would be interesting to measure long term effects by allowing the children to play the games regularly during a few weeks. Bigger and more significant effects might be observed then.

\section{CONCLUSION}

We developed and tested two games that allow children to train their visual perspective taking skills. Besides the motivating environment that every computer game offers, the games offered manipulations of objects in a 3D world. This enabled epistemic actions, which facilitate thinking and problem solving. All children performed well in the games, although there was a large variety in VPT skills among them. A small but significant learning effect was observed, again revealing the potential of tangible games in learning.

\section{ACKNOWLEDGMENTS}

We would like to thank our psychomotor therapist, Sabine Plessers for her valuable input during this study. Her expertise in the domain of visual perspective taking was essential for this work. We are also grateful to the children who participated patiently in this study, and their parents for giving the necessary permissions.

\section{REFERENCES}

[1] Antle, A.N. Exploring how children use their hands to think: An embodied interactional analysis. Behaviour \& Information Technology 32, 9 (2013), 938-954.

[2] Bruner, J.S. Studies in Cognitive Growth. John Wiley \& Sons Inc, 1966. 
[3] Fitzmaurice, G.W. Graspable user interfaces. 1996. http://portal.acm.org/citation.cfm?id=925309.

[4] Flavell, J.H. The development of knowledge about visual perception. Nebraska Symposium on Motivation. Nebraska Symposium on Motivation 25, (1977), 43-76.

[5] Frick, A., Möhring, W., and Newcombe, N.S. Picturing perspectives: development of perspective-taking abilities in 4- to 8-year-olds. Developmental Psychology 5, (2014), 386.

[6] Heller, M.D., Roots, K., Srivastava, S., Schumann, J., Srivastava, J., and Hale, T.S. A Machine Learning-Based Analysis of Game Data for Attention Deficit Hyperactivity Disorder Assessment. Games for Health Journal 2, 5 (2013), 291-298.

[7] Horn, M.S., Crouser, R.J., and Bers, M.U. Tangible Interaction and Learning: The Case for a Hybrid Approach. Personal Ubiquitous Comput. 16, 4 (2012), 379-389.

[8] Hunter, S., Kalanithi, J., and Merrill, D. Make a Riddle and TeleStory: Designing Children's Applications for the Siftables Platform. Proceedings of the 9th International Conference on Interaction Design and Children, ACM (2010), 206-209.

[9] Ishii, H. and Ullmer, B. Tangible bits: towards seamless interfaces between people, bits and atoms. Proceedings of the SIGCHI conference on Human factors in computing systems, ACM (1997), 234-241.

[10] Martin, T. A theory of physically distributed learning: How external environments and internal states interact in mathematics learning. Child Development Perspectives 3, 3 (2009), 140-144.

[11] McNeil, N.M. and Uttal, D.H. Rethinking the use of concrete materials in learning: Perspectives from development and education. Child development perspectives 3, 3 (2009), 137139.
[12] Montessori, M. The montessori method. Transaction Publishers, 2013.

[13] Piaget, J. The Psychology of Intelligence. Routledge, 2001.

[14] Pillias, C., Robert-Bouchard, R., and Levieux, G. Designing Tangible Video Games: Lessons Learned from the Sifteo Cubes. Proceedings of the 32Nd Annual ACM Conference on Human Factors in Computing Systems, ACM (2014), 31633166.

[15] Resnick, M., Martin, F., Berg, R., et al. Digital Manipulatives: New Toys to Think with. Proceedings of the SIGCHI Conference on Human Factors in Computing Systems, ACM Press/Addison-Wesley Publishing Co. (1998), 281-287.

[16] Shaer, O. and Hornecker, E. Tangible User Interfaces: Past, Present, and Future Directions. Found. Trends Hum.Comput. Interact. 3, 1\&\#8211;2 (2010), 1-137.

[17] Surtees, A.D.R., Apperly, I.A., and Samson, D. The use of embodied self-rotation for visual and spatial perspectivetaking. Frontiers in Human Neuroscience 7, (2013), 698.

[18] Vanden Abeele, V., Schutter, B., Geurts, L., et al. P-III: A Player-Centered, Iterative, Interdisciplinary and Integrated Framework for Serious Game Design and Development. In S. Wannemacker, S. Vandercruysse and G. Clarebout, eds., Serious Games: The Challenge. Springer Berlin Heidelberg, 2012, 82-86.

[19] Vandermaesen, M., de Weyer, T., Luyten, K., and Coninx, K. PhysiCube: Providing Tangible Interaction in a Pervasive Upper-limb Rehabilitation System. Proceedings of the 8th International Conference on Tangible, Embedded and Embodied Interaction, ACM (2013), 85-92.

[20] Victor, B. A Brief Rant on the Future of Interaction Design. 2011. http://worrydream.com/ABriefRantOnTheFutureOfInteractio $\mathrm{nDesign} /$. 